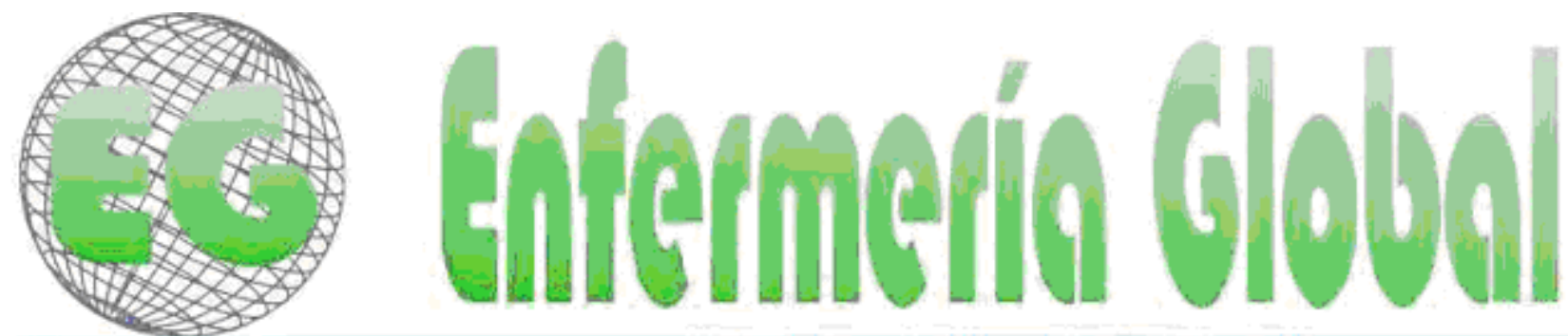

15SN $1696-6+\$ 96$

Reuista electrônica cuatrimestral de Enfermeria

$\mathrm{N}^{\circ} 20$

Octubre 2010

www.um.es/egloball

ADMINISTRACIÓN - GESTIÓN - CALIDAD

\title{
CATETERIZACIÓN VENOSA CENTRAL DE ACCESO PERIFÉRICO MEDIANTE TÉCNICA SELDINGER MODIFICADA EN LA URGENCIA HOSPITALARIA
}

CENTRAL VENOUS CATETHERISATION OF PERIPHERIC ACCESS THROUGH SELDINGER TECHNIQUE MODIFIED IN THE EMERGENCY ROOM

\author{
*Cárcoba Rubio, N., *Ceña Santorcuato, S. \\ *Enfermera. Servicio de Urgencias del Hospital de Cruces. Vizcaya.
}

Palabras clave: Técnica Seldinger modificada, Acceso periférico, Vía venosa central, Cuidados de Enfermería.

Keywords: Modified Seldinger Technique, Peripheral Access, Central Venous Line, and Nursing Care

\section{RESUMEN}

El objetivo de este trabajo es la descripción de la técnica de enfermería para canalización de vía venosa central de acceso antecubital de dos o tres luces hasta vena cava superior, a través de una cánula corta, en condiciones de esterilidad, para la extracción de muestras, administración de medicación endovenosa, nutrición, hemoderivados y monitorización hemodinámica. De este modo, garantizamos la permeabilidad de una vía de acceso, evitando con ello el sufrimiento del paciente por venopunciones continuas.

Para ello, se realizó una revisión y estudio de la bibliografía existente de fuentes de enfermería de urgencias, unidades de intensivos y reanimación. Así como de los protocolos de enfermería de urgencias del Hospital de Cruces.

En concordancia con los resultados encontrados en la literatura y a modo de conclusión, podemos decir que la determinación de protocolos constituye una herramienta de excelencia en los cuidados de enfermería que facilita la toma de decisiones, disminuye la variabilidad, mejora el registro y la satisfacción de profesionales y usuarios.

\section{ABSTRACT}

The aim of this article is the description of the nursing technique of inserting a central catheter by peripheral access to superior vena cava through a short cannula, under sterile conditions, for sample's extraction, administration of intravenous medications, nutrition, blood products, blood collection, haemodinamic monitoring, guaranteeing the permeability of a central line, thereby avoiding the suffering of the patient by venipuncture continuous. For the extraction of samples, administering endovenous medicine, nutrition, hemoderivatives and hemodynamics monitoring. In this way, the 
permeability of a via of access is guaranteed, avoiding the suffering of the patient due to continuous venopuncture.

To that end, we conducted a review and study of the existing literature sources of emergency nursing, intensive and resuscitation units. As well as protocols of emergency nursing of Hospital de Cruces.

In accordance with the results found in literature and in conclusion, we can say that the determination of protocols is a tool for excellence in nursing that facilitates decision-making, reduces variability, and improves registration and satisfaction of professionals and users.

\section{INTRODUCCIÓN}

La canalización de una vía central es un procedimiento clave en el abordaje del enfermo crítico, por lo que, en un intento de minimizar el tiempo de actuación sobre estos pacientes, se ha iniciado su progresiva implantación en el servicio de urgencias.

Como alternativa al catéter de acceso periférico tipo DRUM, que precisa venas de grueso calibre para su canalización, para disminuir los riesgos respecto a la inserción de otros catéteres centrales y teniendo en cuenta el elevado porcentaje de éxito de esta técnica y la comodidad del paciente, nos parece interesante explicar el procedimiento y los cuidados de enfermería que conlleva.

\section{* Definición(2)}

Técnica de canalización percutánea de vía venosa central, a través de un acceso periférico mediante un catéter de doble o triple luz de poliuretano radio opaco, utilizando la técnica de Seldinger modificada, que nos permite:

- Administrar: grandes volúmenes de líquidos, soluciones hipertónicas, soluciones incompatibles a través de luces separadas o varias perfusiones simultáneamente.

- Monitorización hemodinámica.

- Extracción muestras sanguíneas.

* Personal necesario(1)

1 enfermera y 1 auxiliar

\section{* Ámbito de aplicación(1)}

Un box de urgencias.

\section{* Material necesario(2)(5)(8)}

- Mesa auxiliar

- Cepillo con antiséptico

- Empapador

- Compresor

- Antiséptico al uso: povidona iodada o clorhexidina $2 \%$.

- Gasas estériles

- 1 paño estéril y 1 paño fenestrado

- Bata y guantes estériles

- Gorro y mascarilla

- Anestésico tópico (EMLA® o Cloretiloß)

- 2 jeringas de $10 \mathrm{ml}$ 
- Abocath $\AA$ no 18

- Catéter de doble/triple luz(Arrow® de $60 \mathrm{~cm})$

- 2 sueros, sistemas y llaves de 3 vías.

- Adhesivo para fijar el catéter (steri-strip®)

- Apósito estéril transparente

\section{DESCRIPCIÓN DE LA TÉCNICA (2)(4)(6)}

\section{* Preparación del paciente}

> Informar adecuadamente al paciente de la técnica que se le va a realizar. Esto nos ayudará a lograr su colaboración durante la realización de la misma.

> Monitorizar el electrocardiograma antes, durante y después del procedimiento. Es muy importante una adecuada visualización, ya que durante la introducción, tanto de la guía como del catéter, se debe vigilar la aparición de arritmias. Registrar constantes vitales.

$>$ Colocar al paciente en posición decúbito supino o Trendelemburg, según nos permita la patología del paciente.

> Valorar las venas de ambas extremidades superiores en la fosa antecubital. Se elegirá preferentemente el brazo derecho y las venas basílica, mediana y cefálica, en este orden, puesto que la vena basílica del brazo derecho es la de acceso más directo anatómicamente a la aurícula derecha, y por tanto, la introducción del catéter será presumiblemente más sencilla.

$>$ Se pueden emplear dos tipos de anestésicos locales, EMLA $\AA^{\circ}$ o Cloretilo ${ }^{\circledR}$. El Cloretilo ${ }^{\circledR}$ es de acción inmediata, pero si se elige EMLA ${ }^{\circledR}$, se debe aplicar la pomada sobre la zona seleccionada entre 30 y 60 minutos antes de la venopunción, retirándola justo antes de aplicar el antiséptico.

> Aplicar el antiséptico de forma circular de dentro hacia fuera. La Clorhexidina $2 \% \AA$ tarda medio minuto en secarse, y la povidona yodada 2 minutos. En caso de tener que actuar con celeridad, se puede secar con gasas estériles, para evitar que el antiséptico penetre en el torrente sanguíneo.

\section{Preparación del personal}

Utilizar técnica estéril en todo momento. Esto implica que la enfermera/o responsable del procedimiento realizará lavado quirúrgico de sus manos, previa colocación de mascarilla y gorro. El secado de las manos se hará con compresas estériles y se colocará bata estéril.

\section{* Realización de la técnica}

Dolocar un empapador bajo el brazo elegido.

> Preparar una mesa auxiliar con paños estériles y todo el material necesario. Aprovechar para revisar que no falta nada en el equipo de vía central. 


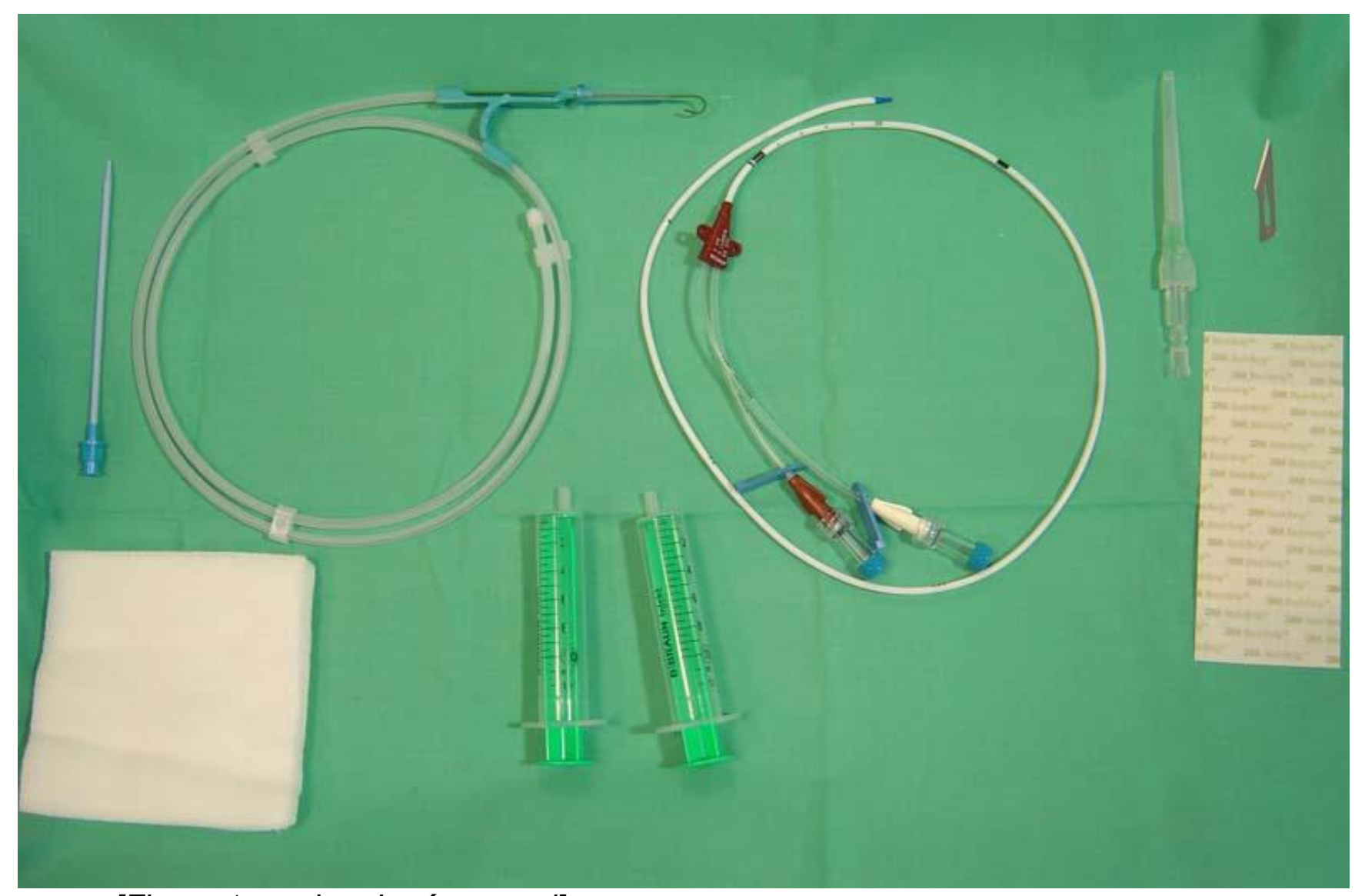

[Figura 1, equipo de vía central]

> Colocar paño fenestrado sobre el brazo y ampliar el campo estéril con otro paño, puesto que la longitud del catéter y de la guía hace que sea más complicado que no se salgan del campo.

$>$ Canalizar vena mediante un Abocath ${ }^{\circledR}$. El mínimo será del $n^{\circ} 18$, ya que si es de menor grosor, no pasará la guía a través de su luz. Al contrario, si es un no 14 o 16, facilitarán la posterior introducción del catéter, ya que actuarán como dilatadores.

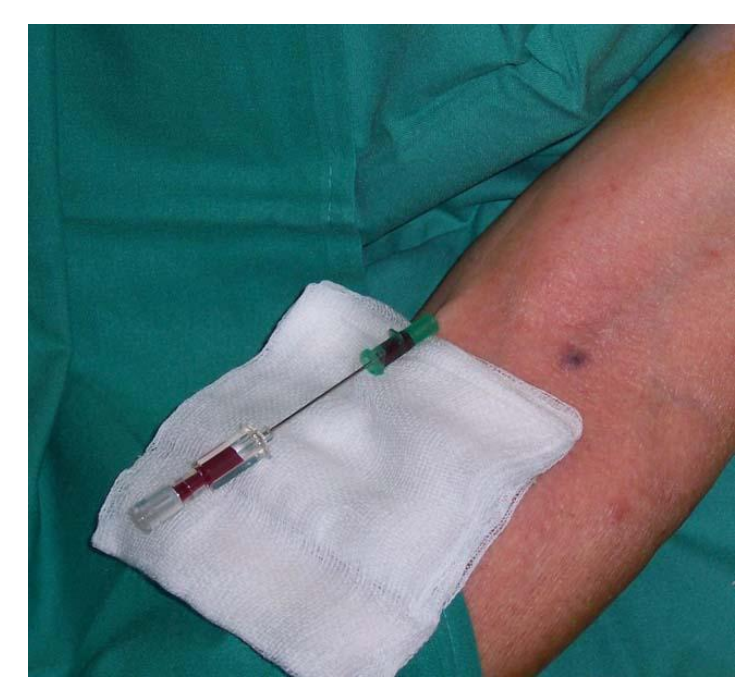

[Figura 2, colocación del catéter]

> Retirar el compresor para permitir la progresión de la guía (personal no estéril) 
> Pedir la colaboración del paciente, haciendo que gire la cabeza hacia el lado de la punción, y empezar a introducir la guía a través del angiocatéter. La guía se presenta con la punta blanda, de forma curva, fuera de su introductor, por lo que se moviliza con el pulgar hasta que el final de la guía coincida con el extremo de plástico que se adaptará al angiocatéter. Después se introducirá con suavidad. Si se nota dificultad, se retrocede unos centímetros, se moviliza el brazo y se reintenta.

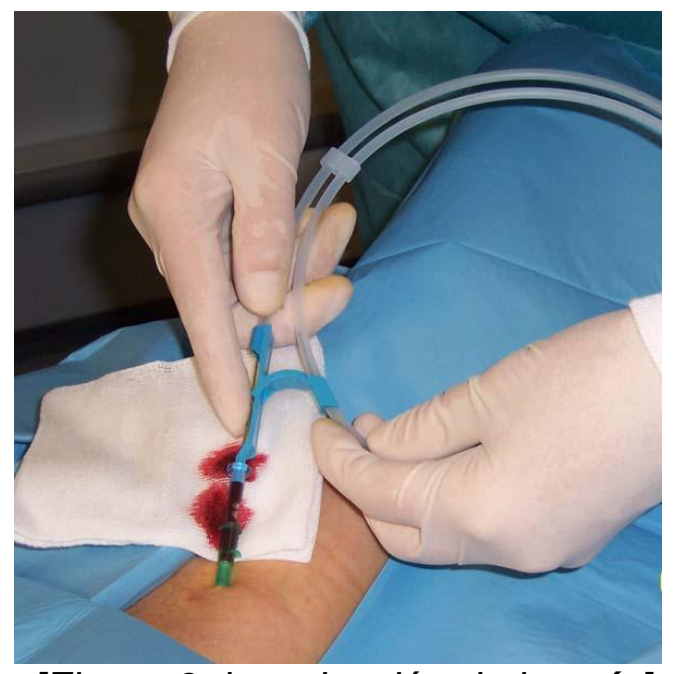

[Figura 3, introducción de la guía]

> Vigilar el electrocardiograma en todo momento. Si aparecen arritmias, se retira la guía hasta que vuelva a la situación normal del paciente.

$>$ Dejar fuera suficiente porción de guía como para poder manejarla con seguridad, y sacar el angiocatéter a través de la guía.

$>$ Deslizar el dilatador a través de la guía. Al traspasar el tejido subcutáneo y la piel, se debe ejercer cierta fuerza. Si fuera necesario, se puede realizar un corte con un bisturí, en la zona de la punción, colocando el filo hacia arriba. Basta con introducir la punta del dilatador, puesto que en el brazo las venas están muy superficiales.

$>$ Retirar el dilatador y aplicar presión con una gasa sobre el punto de inserción.

$>$ Retirar el tapón de la luz distal del catéter, ya que la guía saldrá por ese punto, y empezar a introducir el catéter deslizándolo por la guía. No introducir el catéter en la vena hasta que la guía salga unos centímetros por la luz distal.)

$>$ Mantener siempre sujeta la guía mientras se introduce el catéter suavemente.

> Introducir hasta $40-45 \mathrm{~cm}$, y teniendo en cuenta que si el brazo es el izquierdo, unos centímetros más. El catéter tiene medidas en su longitud final

$>$ Retirar la guía con cuidado y comprobar el reflujo de sangre en las dos luces con jeringas de $10 \mathrm{ml}$, conectando después los equipos de suero previamente purgados en cada luz.

\section{* Fijación del catéter}

$>$ Retirar la sangre del brazo y zona de punción con suero fisiológico, y posteriormente aplicar el antiséptico seleccionado, clorhexidina $2 \%$ o povidona iodada.

> Para fijar el catéter se puede emplear Steri-strip®, colocando una corbata alrededor del catéter, sin tapar el punto de inserción, más un apósito oclusivo estéril. 


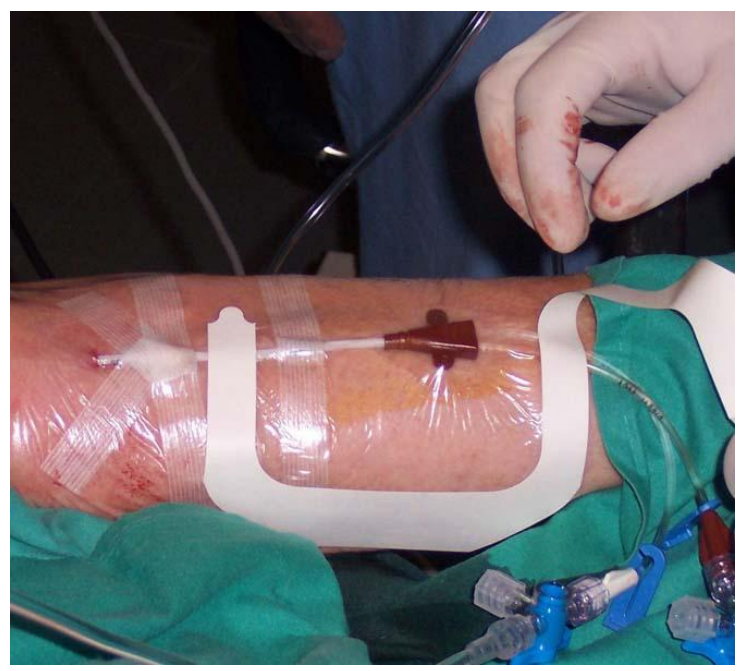

[Figura 4, fijación del catéter]

Retirar el material punzante y depositarlo en el contenedor adecuado.

- Quitar el campo estéril, dejar al paciente en la posición más cómoda posible, y lavarse las manos.

\section{COMPLICACIONES POTENCIALES (2) (7)}

* Existen de dos tipos:

$*$

\section{Relacionadas con la punción:}

- Extrasístoles o arritmias: desencadenadas al introducir la guía. Generalmente se resuelven espontáneamente al retirar ésta.

- Sangrado o hematoma: se resuelven al comprimir la zona.

- Embolia gaseosa: riesgo inherente a toda punción. Para intentar evitarlo se debe purgar bien todo el catéter y realizar la punción en posición en Trendelenburg.

- Lesiones nerviosas: por punción directa en nervio o compresión de un hematoma.

\section{Relacionadas con el catéter:}

- Infección: es la más frecuente y está en relación con el tiempo de uso y la manipulación. Incidencia 20 al $60 \%$ y llega a producir bacteriemia en el $10 \%$ de los casos.(8)

- Trombosis: existen factores que aumentan el riesgo de trombosis:

- 2 o más punciones

- El tipo de líquido infundido (más frecuente en nutrición parenteral)

- Estado de hipercoagulabilidad.

- Obstrucción: puede ser total o parcial (imposibilidad de extraer o infundir) o parcial (imposibilidad de extraer) se produce por acúmulo de fibrina.

- Desplazamiento accidental del catéter

- Rotura del catéter. 


\section{INTERVENCIONES ENFERMERAS DE VIGILANCIA Y CONTROL}

Controles (1) (2)

$>$ Seguir las recomendaciones de la Sociedad Española de Medicina Intensiva, Crítica y Unidades Coronarias (SEMICYUC) (9), para disminuir la aparición de las complicaciones:

- Valorar la indicación de la inserción de un catéter venoso central.

- Reconocimientos de factores de riesgos que puedan dificultar la canalización, tales como índice de masa corporal, obesidad, ventilación mecánica, arterioesclerosis grave, sepsis, arritmias ventriculares, EPOC, antecedentes de radioterapia, etc.

- Colocación o supervisión por personal experto si se prevé dificultad.

- Elección del sitio de inserción.

- No realizar más de dos o tres intentos de venopunción.

- No practicar cambios rutinarios de catéter para prevenir la infección.

- Comprobación de la posición correcta del catéter mediante Rx de tórax. La posición más adecuada es en vena cava superior, tercer espacio intercostal.

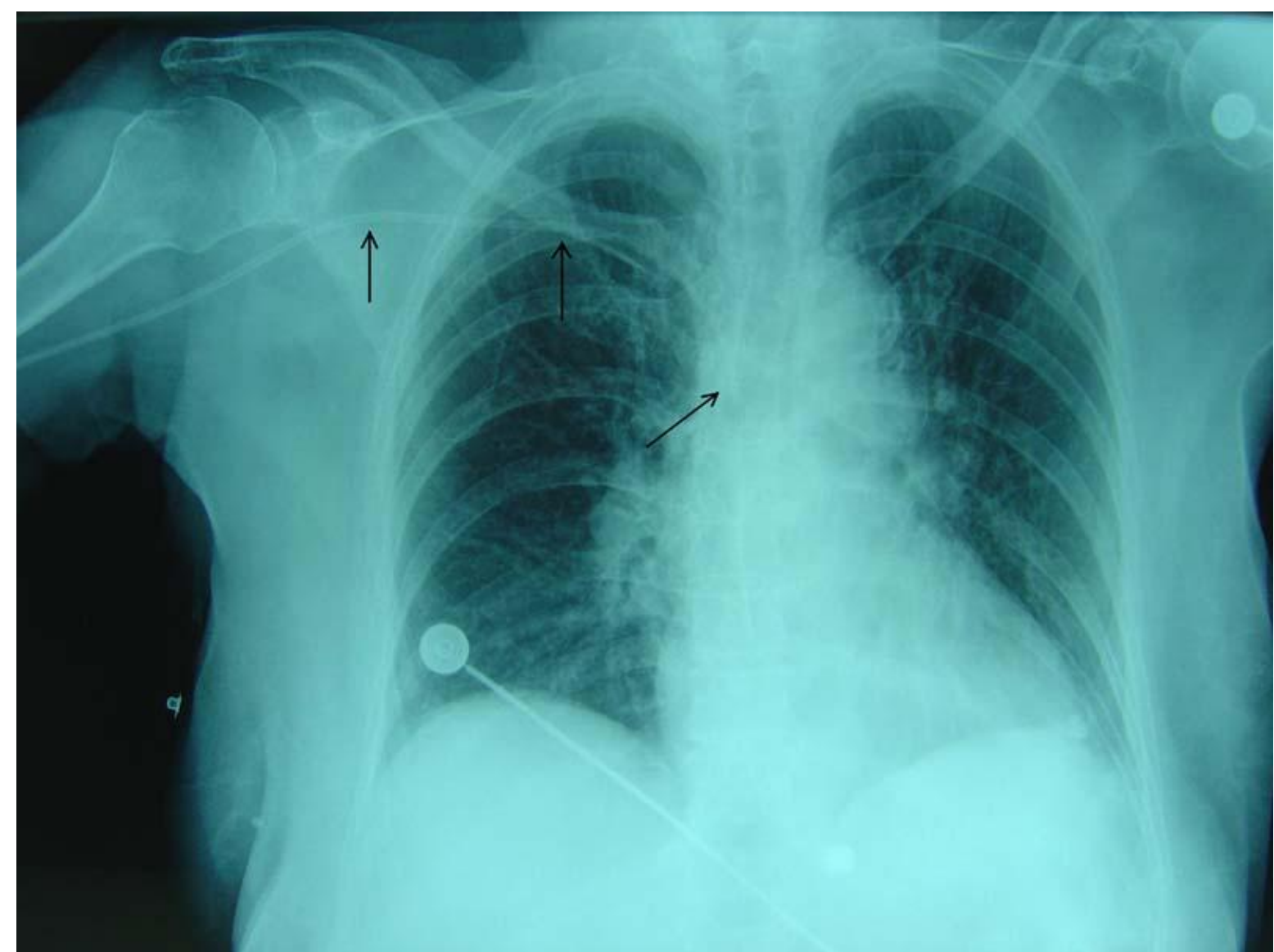

[Figura 5, comprobación radiológica]

- Colocación y retirada del catéter en Trendelemburg.

- Oclusión de la luz de la aguja durante la inserción.

Verificar funcionamiento adecuado de las perfusiones.

> Control de las complicaciones relacionadas con la punción o con el catéter.

$>$ Valorar diariamente la indicación del mantenimiento del catéter venoso central. 


\section{Registro y observaciones de enfermería (1)}

Registro de todos los datos relacionados con el acceso y catéter en la gráfica de enfermería de urgencias:

$>$ Tipo de catéter y no luces

Extremidad y vena de acceso

$>$ Fecha de acceso

$>$ Incidencias que surjan durante la inserción en el evolutivo de enfermería-

\section{BIBLIOGRAFÍA}

1. Cateterización venosa central a través de acceso periférico antecubital. Recomendaciones para UCI y Urgencias del Hospital de Cruces. Osakidetza. Versión: enero 2008.

2. Oliva Cesar O, Suriana Jiménez R, González Caro JM, Rodríguez Flores R. Cuidados de enfermería en el cateterismo venoso central de acceso periférico con catéter de doble luz multilumen mediante técnica de Seldinger. Nure Investigación, no 29, julioagosto 07.

3. García Velasco, Sánchez. Morago S, Sánchez Coello Ma․ Inserción de un catéter central periférico. Un procedimiento de enfermería. Rev. Metas Enf. 2201; 38:12-15.

4. Martos Arroyo B, Arza Bilbao A, Revilla Domínguez C. La enfermería ante la infección de catéteres venosos centrales. Revista Oiñarri, Septiembre 2007, número 52.

5. Nursing Procedure Manual of University of North Carolina Hospitals. Disponible en: http://www.unchealthcare.org/site/Nursing/nurspractice/procedures/procedures/proced urep12.pdf/

6. Driscoll M, Buckenmyer Ch, Spirk M, Molchany C Inserting and mantainig peripherically inserted central catheters. Mednursing. Diciembre 1997. Disponible en:http://findarticles.com/p/articles/mi m0FSS/is n6 v6/ai n18607581

7. Williams B, Consentido RN. Insertion of PICC Lines- Using MST. Nursing Spectrum. Marzo 2003.

Disponible en:http://include.nurse.com/apps/pbcs.dll/article?AID=2003303240346

8. Centers for Disease Control and Prevention. Guidelines for the prevention of intravascular catheterrelated infections. MMWR 2002; 5:1-29.

9. Diez recomendaciones para prevenir las complicaciones asociadas a la colocación de catéteres venosos. Disponible en:

http://www.medicinadigital.com/index2.php?option=com content\&do $\mathrm{pdf}=1 \& \mathrm{id}=4373$

ISSN 1695-6141

(C) COPYRIGHT Servicio de Publicaciones - Universidad de Murcia 